\title{
Skin test activity of an antigen fraction prepared from Mycobacterium leprae compared with standard lepromin and tuberculin PPD in leprosy patients
}

\author{
LIV J REITAN*§, ELS M J TOUW-LANGENDIJK†, \\ O CLOSS $\ddagger \&$ A BELEHU* \\ * Armauer Hansen Research Institute (AHRI), PO Box 1005, Addis \\ Ababa, Ethiopia; $\dagger$ All Africa Leprosy and Rehabilitation Training \\ Centre (ALERT), PO Box 165, Addis Ababa, Ethiopia; and \\ $\ddagger$ University of Oslo, Institute for Experimental Medical Research, \\ Ulleval Hospital, Oslo, Norway
}

Accepted for publication 11 July 1983

\begin{abstract}
Summary The reactivity of a cell wall antigen fraction, MLW1, prepared from Mycobacterium leprae, which induces strong lymphocyte responses in vitro, was compared in the lymphocyte stimulation test (LST) and the 48-hr skin test reaction in leprosy patients. A strong LST response was usually accompanied by a strong skin test response and vice versa. As a skin test reagent MLWl was compared with standard lepromin and tuberculin PPD, and a significant correlation $(r=0.79, p<0.001)$ was found between MLWl and standard lepromin. Being a purified and highly-active preparation that can be standardized based on protein concentration, MLW 1 should be considered as an alternative to lepromin in the early reaction.
\end{abstract}

\section{Introduction}

The standardization of lepromin is based on the number of acid fast bacilli $/ \mathrm{ml} .^{1}$ In contrast, the standardization of tuberculin PPD is based on functional activity assayed in experimental animals. Another disadvantage of lepromin compared to tuberculin, which has been shown in both experimental animals ${ }^{2}$ and $\operatorname{man}^{3,4}$, is that repeated testing leads to sensitization of the test subject. ${ }^{5}$ A skin test reagent which does not induce sensitization and is easy to standardize, like tuberculin, would be a great improvement in leprosy.

A cell wall antigen preparation from Mycobacterium leprae called MLW1,

$\S$ Correspondence: L J Reitan, Institute of Immunology and Rheumatology, Fr. Qvamsgt. 1, Oslo 1, Norway. 
producing only one line corresponding to $M$. leprae antigen 7 in crossed immunoelectrophoresis, induced strong in vitro lymphocyte responses in patients with tuberculoid leprosy and in healthy contacts of leprosy patients, while lymphocytes from lepromatous leprosy patients and non-exposed controls did not respond. ${ }^{6}$ Like PPD, MLW1 also consists of solubilized antigens in which the amount of protein can be determined. Thus, it can be standardized on the basis of biological activity per $\mu \mathrm{g}$ protein as PPD for use as a skin test reagent. However, since MLW1 is known to contain crossreacting antigenic determinants, a marked contribution of such determinants to the skin test activity of the preparation would reduce its usefulness as a skin test reagent.

In the present study the ability of MLW1 to stimulate lymphocytes in vitro was compared with its skin test activity in leprosy patients. Its potency and specificity as a skin test reagent were compared with standard lepromin and tuberculin PPD.

\section{Materials and methods}

\section{PATIENTS}

All the patients in this study attended the All Africa Leprosy and Rehabilitation Training Centre (ALERT) in Addis Ababa, Ethiopia. Thirty-four patients, 16 females and 18 males, with a median age of 24 years (range 13 to 39), were included. They were all classified clinically according to the Ridley-Jopling scale, ${ }^{7}$ and, in addition, 13 patients were also classified histologically. There were two patients with tuberculoid/borderline tuberculoid leprosy (TT/BT), 21 with BT, 6 with borderline lepromatous (BL) and 5 with lepromatous leprosy (LL). Ten of the patients had been treated with dapsone (DDS, $100 \mathrm{mg} /$ day) for a period varying from 1 month to 5 years; the others were untreated.

\section{SKIN TESTING}

The following three antigens were injected intradermally on the same forearm with the injection sites at least $4 \mathrm{~cm}$ apart in a volume of $0.1 \mathrm{ml}: 1$ The lepromin (obtained from Dr W F Kirchheimer, Carville, Louisiana) was armadillo derived and contained $1.6 \times 10^{7}$ bacilli. 2 A fractionated preparation of $M$. leprae of armadillo origin, called $\mathrm{MLW} 1^{6}, 0 \cdot 2$ or $2 \mu \mathrm{g}$ protein. 3 Tuberculin purified protein derivative (PPD) from Statens Seruminstitut, Copenhagen, Denmark, Batch RT23, $0.04 \mu$ g protein (2 TU). Reactions were read after $48 \mathrm{hr}$; the induration was measured with a ruler and the mean of the longitudinal and transverse diameter recorded. An induration of $5 \mathrm{~mm}$ or more was considered a positive reaction. 


\section{LYMPHOCYTE STIMULATION TEST}

Blood was drawn for the LST on the same day as skin testing was performed, but prior to the injection of the antigen. Mononuclear cells were isolated and cultured as previously described. ${ }^{6}$ Briefly, $10^{5}$ cells/well were stimulated with $1 \mathrm{MLW} 1$, the same preparation as used in skin testing, and 2 tuberculin PPD, Batch RT33, Statens Seruminstitut, and then cultured in triplicates for 6 days. Proliferation was measured as ${ }^{3} \mathrm{H}$-thymidine incorporation. The median counts per minute (cpm) for each triplicate was used and the degree of stimulation expressed as $\Delta \mathrm{cpm}=\mathrm{cpm}$ of stimulated culture $-\mathrm{cpm}$ of unstimulated control. According to our previous study ${ }^{8}$ an individual with an LST response of $\Delta \mathrm{cpm} \geqq 5000$ was defined as a responder.

\section{Results}

The antigen preparation MLW1 was tested as a skin test reagent in patients with various clinical forms of leprosy at two doses, either 0.2 or $2 \mu \mathrm{g}$ per skin test site. Figure 1 compares in 23 patients the capacity of MLW1 to induce a 48-hr skin reaction with its capacity to stimulate lymphocytes in vitro $(r=0 \cdot 66, p<0 \cdot 001)$ at a concentration of $0 \cdot 1 \mu \mathrm{g} / \mathrm{ml}$ which was shown before to give the highest median response in the BT group. ${ }^{6}$ In 11 of the patients a positive LST response was accompanied by a positive skin test response and vice versa. Five patients (1

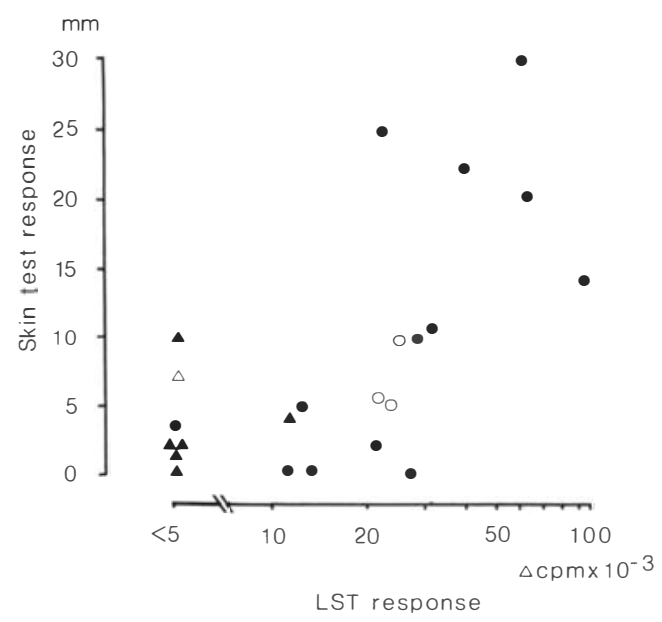

Figure 1. In vitro lymphocyte responses to $0 \cdot 1 \mu \mathrm{g} / \mathrm{ml}$ and skin test responses to $0 \cdot 2 \mu \mathrm{g}(O, \Delta)$ a nd $2 \mu \mathrm{g}$ $(\bullet, \Delta)$ of MLW1 in patients with various clinical forms of leprosy (TT/BT, n=2; BT n=14;, 0$)$, $(\mathrm{BL}, \mathrm{n}=4 ; \mathrm{LL}, \mathrm{n}=3 ; \boldsymbol{\Delta}, \Delta)$. Lymphocyte stimulation is shown as net counts per minute $(\Delta \mathrm{cpm}=\mathrm{cpm}$ of stimulated culture $-\mathrm{cpm}$ of unstimulated control) after incorporation of ${ }^{3} \mathrm{H}$-thymidine, and skin test activity as diameter of induration in $\mathrm{mm}$ read at $48 \mathrm{hr}$. 
TT/BT, $3 \mathrm{BT}$; $1 \mathrm{BL})$ with moderately strong LST responses $(11,000 \Delta \mathrm{cpm}$ to $27,000 \Delta \mathrm{cpm}$ ) showed a negative skin test response, while two patients (2 BL) with moderate skin test response $(7$ and $10 \mathrm{~mm}$ ) showed very weak LST responses, $2300 \Delta \mathrm{cpm}$ and $1500 \Delta \mathrm{cpm}$, respectively. In the 16 patients (2TT/BT, $13 \mathrm{BT}, 1$ $\mathrm{BL})$ who were responders in the LST $(\Delta \mathrm{cpm} \geqq 5000)$ the strength of the responses correlated better $(r=0 \cdot 70, p<0 \cdot 01)$ than in the 13 patients $(2 \mathrm{TT} / \mathrm{BT}, 9 \mathrm{BT}, 1 \mathrm{BL}$, $1 \mathrm{LL})$ who showed a positive skin test response $(\geqq 5 \mathrm{~mm})(r=0 \cdot 50, p<0 \cdot 10)$.

In 14 of the patients with TT/BT and BT leprosy, the skin test and LST were performed with both MLW1 and PPD, and in Figure 2(A) and (B), the potencies of these two reagents are compared in both tests. Based on the protein concentrations which were used, the ratio between the doses of MLW1 and PPD was 1:10 in the LST and 50:1 in the skin test. There were three patients who showed stronger responses to MLW1 than to PPD in the LST (Figure 2(A)), and two of these were among the seven patients (with a positive skin test response to MLW1) who showed stronger skin test responses to MLW 1 than to PPD (Figure 2(B)), showing that antigenic specificity was expressed in both tests.

In Figure 3 skin test activity of standard lepromin and MLW1 is compared in patients throughout the leprosy spectrum. Fourteen of the patients were tested with a dose of $0.2 \mu \mathrm{g}$ MLW1 per skin test site, and three of these were negative to MLW1 and positive to lepromin. The remaining 20 patients were tested with a dose of $2.0 \mu \mathrm{g} \mathrm{MLW} 1$, and three of these were positive to MLW1 and negative to lepromin. A dose of MLW1 between $0 \cdot 2$ and $2 \cdot 0 \mu \mathrm{g}$ appeared to correspond in
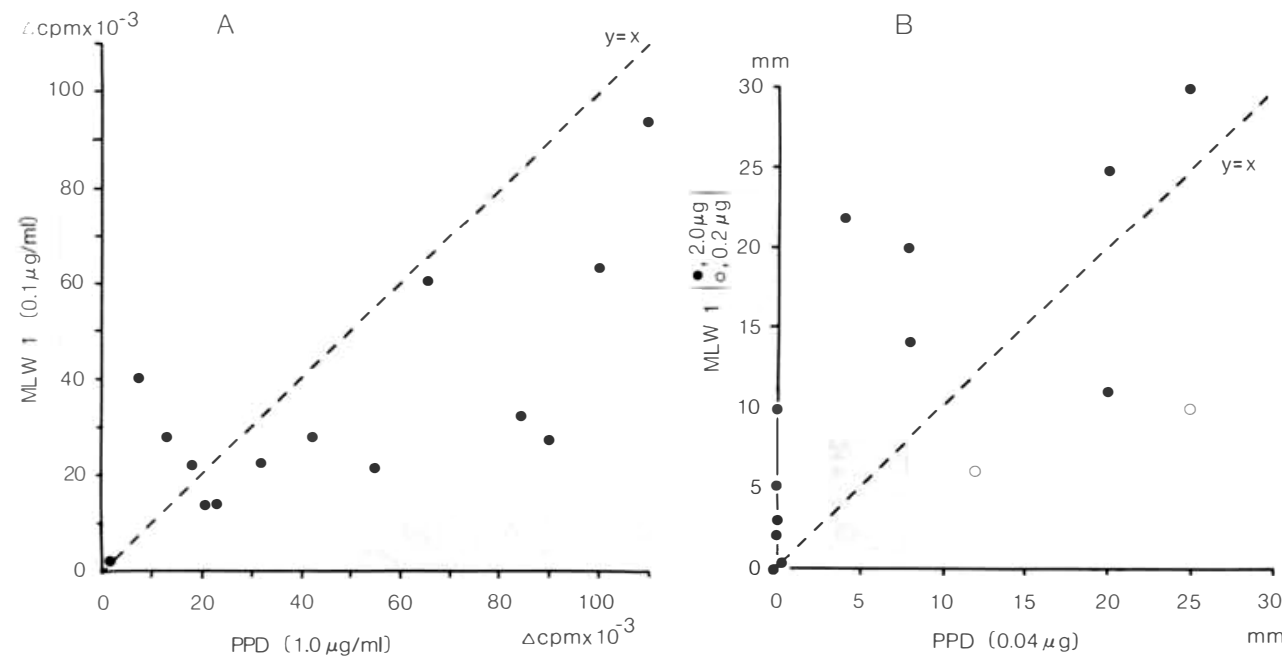

Figure 2. In vitro lymphocyte stimulation (A) and skin test activity (B) in 14 patients with TT/BT and BT leprosy. They were skin tested with a dose of $2 \mu \mathrm{g}$ of MLWl $(\bullet)$ except for two patients who were tested with $0 \cdot 2 \mu \mathrm{g}(0)$, while the strength of PPD was $2 \mathrm{TU}(0 \cdot 04 \mu \mathrm{g})$. The lines of identity, $\mathrm{y}=\mathrm{x}$, are stippled. For further explanation see legend to Figure 1. 


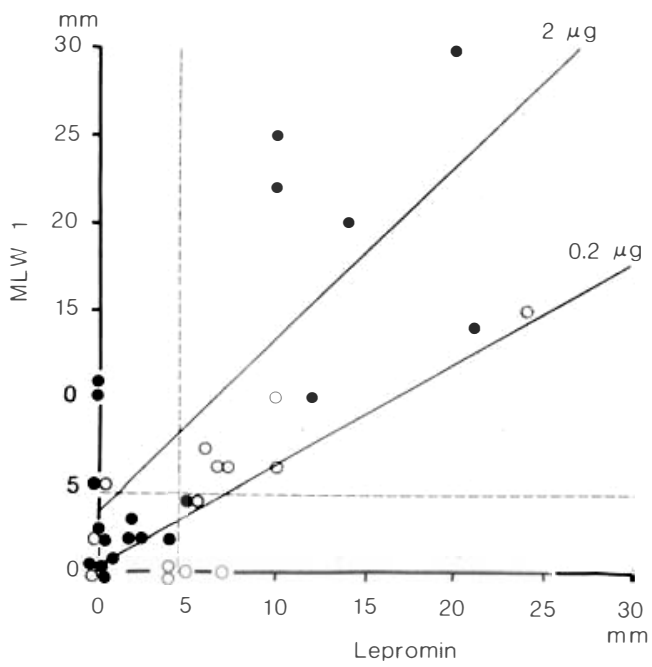

Figure 3. Correlation plot of skin test activities with $\mathrm{MLW} 1(2 \mu \mathrm{g}, \bullet ; 0 \cdot 2 \mu \mathrm{g}, \mathrm{O})$ and lepromin in 34 patients with various clinical forms of leprosy (TT/BT, $n=2 ; \mathrm{BT}, \mathrm{n}=21 ; \mathrm{BL}, \mathrm{n}=6 ; \mathrm{LL}, \mathrm{n}=5$ ). The lines of regression at both doses are solid and the dividing lines between positive and negative responses are stippled. For further explanation see legend to Figure 1.

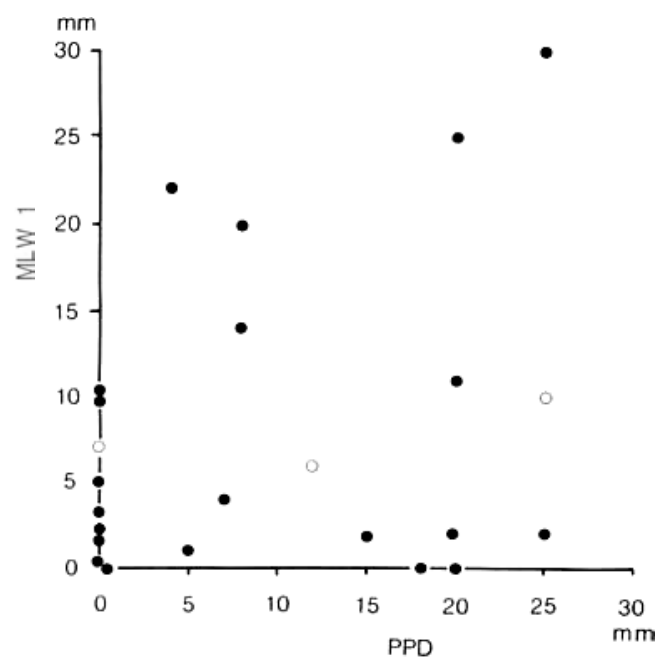

Figure 4. Correlation plot of skin test activities with MLWl $(2 \mu \mathrm{g}, \bullet ; 0 \cdot 2 \mu \mathrm{g}, \mathrm{O})$ and tuberculin PPD, $2 \mathrm{TU}$, in 24 leprosy patients (TT/BT, $\mathrm{n}=2 ; \mathrm{BT}, \mathrm{n}=14 ; \mathrm{BL} ; \mathrm{n}=5 ; \mathrm{LL}, \mathrm{n}=3$ ). For further explanation see legend to Figure 1. 
strength to standard lepromin. A correlation was found in skin test activity between lepromin and MLW1 $(r=0 \cdot 79, p<0 \cdot 001)$.

Previously, we demonstrated ${ }^{6}$ that tuberculin PPD and MLW1 show different antigenic specificities in the LST, and in the present study no correlation $(r=0 \cdot 26)$ was found between MLW1 and tuberculin PPD in the 24 patients tested with both antigens (Figure 4). Apparently, antigenic determinants which may be common to MLW1 and PPD did not greatly influence the skin test activity of MLW1.

\section{Discussion}

Using various antigens, skin test and LST responses have been reported by many workers to correlate well, ${ }^{9-14}$ and soluble antigens have given better correlations than particulate antigens. ${ }^{15}$ In leprosy, a crude antigen consisting of $M$. leprae bacilli of either human or armadillo origin has been most commonly used both in the skin test and the LST assays. Leprosin A, a skin test reagent made by Stanford et al. ${ }^{16}$ is a total sonicate of the bacilli, and since it consists of solubilized antigens, its protein content can be determined. But, in contrast to the MLW1 preparation which contains mainly one antigenic component, Leprosin A consists of various antigenic components. The MLW 1 preparation has previously been shown to be a particularly potent stimulator in the LST. ${ }^{6}$ Looking at individual responses both in leprosy patients and healthy contacts of leprosy patients, they were higher for MLW1 than for a preparation of whole $M$. leprae bacilli of human origin. ${ }^{8}$ In the present study we have compared the potency of MLW1 to stimulate lymphocytes in vitro with its potency to induce an early skin reaction in patients with leprosy. It seems that the MLW1 preparation can also induce a fairly strong reaction in patients with a positive LST response. The finding of a negative skin test response in some of the patients with a moderate to strong LST response is in agreement with others who reported the LST to be more sensitive than the skin test in tuberculosis. ${ }^{17-19}$

Antibodies to the ML7 antigen have been shown to cross-react extensively with the BCG60 antigen, ${ }^{20}$ and these antigens appear to be the major constituents of the MLW1 preparation and tuberculin PPD, respectively. When the LST responses to MLW1 of healthy contacts of leprosy patients and of non-exposed controls were compared, they were completely separated, ${ }^{6}$ and all the individuals in these two groups showed higher responses to PPD than to MLW1, in contrast to the group of patients with TT and BT leprosy where one third showed higher responses to MLW1 than to PPD. ${ }^{21}$ In spite of the extensive cross-reaction which may be expressed to a varying extent in the individual responses, these results show that MLW1 and PPD are recognized as different antigens. With regard to the comparisons of the skin test and LST responses in the present study, it seems that the different antigenic specificity of MLW1 and PPD can be expressed in the skin test results, even though the relative doses of the two antigens used in these two tests are not directly comparable. 
Because of the known cross-reactivity with other mycobacterial antigens, the MLW1 preparation should not be regarded as a specific reagent in tests for DTH to $M$. leprae until it has been further tested. However, one may compensate for a certain lack of specificity by using MLW1 in combination with other antigens like tuberculin PPD and looking at the relative responses in the skin test and LST. ${ }^{6}$ Further elucidation of its specificity is needed to evaluate its role as a prospective skin test reagent in leprosy.

\section{Acknowledgments}

This work was supported by the Norwegian and Swedish Save the Children Organizations, the Norwegian Agency for International Development (NORAD), the Immunology of Leprosy Project (IMMLEP) component of the WHO/UNDP/World Bank Special Programme for Research and Training in Tropical Diseases, and Anders Jahre's Fund for the Promotion of Science.

We are grateful to Dr Jan Warndorff, ALERT, for the histological examination of the biopsies.

\section{References}

${ }^{\prime}$ Hanks JH, Abe M, Nakayama T, Tuma M, Bechelli LM, Martinez Dominguez V. Studies towards the standardization of lepromin. Progress and Prospects. Bull Wld Hlth Org, 1970; 42: 703 .

2 Azulay RD. Lepromin retesting as a factor of lepromin test positivation. Int J Lepr, 1974; 42: 428.

${ }^{3}$ Bechelli LM. The influence of repeated lepromin testing on the Mitsuda reaction in healthy people. Int J Lepr, 1959; 27: 228.

${ }^{4}$ Lara CB. Mitsuda's skin reaction (lepromin test) in children of leprous parents. II. Observations on newly-born to eighteen-month-old children. Int J Lepr, 1940; 8: 15.

${ }^{5}$ Keystone EC, Demerieux P, Gladman D, Poplonski L, Piper S, Buchanan R. Enhanced delayed hypersensitivity skin test reactivity with serial testing in healthy volunteers. Clin exp Immunol, 1980; 40: 202.

${ }^{6}$ Closs O, Reitan LJ, Negassi K, Harboe M, Belehu A. In vitro stimulation of lymphocytes in leprosy patients, healthy contacts of leprosy patients and subjects not exposed to leprosy. Comparison of an antigen fraction prepared from Mycobacterium leprae and tuberculin purified protein derivative. Scand J Immunol, 1982; 16: 103.

7 Ridley DS, Jopling WH. Classification of leprosy according to immunity. A five-group system. Int J Lepr, 1966; 34: 255.

${ }^{8}$ Reitan LJ, Closs O, Belehu A. In vitro lymphocyte stimulation in patients with lepromatous and borderline tuberculoid leprosy. The effect of dapsone treatment on the response to Mycobacterium leprae antigens, tuberculin purified protein derivative and non-mycobacterial stimulants. Int J Lepr, 1982; 50: 455.

9 Cox RA, Lundberg DI, Arnold DR. Lymphocyte transformation assays as a diagnostic tool in tuberculosis of children. Am Rev Respir Dis, 1981; 123: 627. 
${ }^{10}$ Fleer A, van der Hart M, Block-Schut BJT, Schellekens PTA. Correlation of PPD and BCG-induced leukocyte migration inhibition, delayed cutaneous hypersensitivity, lymphocyte transformation in vitro and humoral antibodies to PPD in man. Eur J Immunol, 1976; 6: 163.

11 Hinz, CF, Daniel TM, Baum GL. Quantitative aspects of the stimulation of lymphocytes by tuberculin purified protein derivative. Int Arch Allergy, 1970; 38: 119.

12 Kerby GR. Correlation of tuberculin skin reaction with in vitro lymphocyte transformation. Am Rev Respir Dis, 1968; 97: 904.

13 McFarland W, Heilman DH. Comparison of lymphocyte transformation and intradermal reactions to tuberculins. Am Rev Respir Dis, 1966; 93: 742.

14 Senyk G, Hadley WK. In vitro correlates of delayed hypersensitivity in man: ambiguity of polymorphonuclear neutrophils as indicator cells in leukocyte migration test. Infect Immun, 1973; 8: 370.

15 Räsänen L. Skin test reactivity and in vitro responses to microbes and microbial antigens. Clin exp Immunol, 1980; 40: 566.

16 Stanford JL, Rook GAW, Samuel N, Madlener F, Khamenei AA, Nemati T, Modabber F, Rees RJW. Preliminary immunological studies in search of correlates of protective immunity carried out on some Iranian leprosy patients and their families. Lepr Rev, 1980; 51: 303.

17 Matsaniotis N, Tsenghi C, Economou-Mavrou C, Metaxotou-Stavridaki C. Skin hypersensitivity and in vitro lymphocytic reactivity to tuberculin in childhood. $J$ Pediatr, 1968; 72: 599.

18 Miller SD, Jones HE. Correlation of lymphocyte transformation with tuberculin skin-test sensitivity. Am Rev Respir Dis, 1973; 107: 530.

${ }^{19}$ Smith JA, Reichman LB. Lymphocyte transformation. An aid in the diagnosis of tuberculosis in patients with nonreactive skin tests. Am Rev Respir Dis, 1972; 106: 194.

${ }^{20}$ Harboe M, Closs O, Bjorvatn B, Kronvall G, Axelsen NH. Antibody response in rabbits to immunization with Mycobacterium leprae. Infect Immun, 1977; 18: 792.

${ }^{21}$ Closs O, Reitan LJ. In vitro lymphocyte stimulation using a purified antigen of Mycobacterium leprae and tuberculin PPD. Lepr Rev, 1981; 52, Suppl. 1:251. 